This report was prepared as an account of work sponsored by an agency of the United States Government. Neither the United States Government nor any agency thereof, nor any of their employees, makes any warranty, express or implied, or assumes any legal liability or responsibility for the accuracy, completeness, or usefulness of any information, apparatus, product, or process disclosed, or represents that its use would not infringe privately owned rights. Reference herein to any specific commercial product, process, or service by trade name, trademark, manufacturer, or otherwise does not necessarily constitute or imply its endorsement, recommendation, or favoring by the United States Government or any agency thereof. The views and opinions of authors expressed herein do not necessarily state or reflect those of the United States Government or any agency thereof.

\title{
LATTICE CALCULATIONS OF ELECTROWEAK DECAY AMPLITUDES
}

\author{
Stephen R. Sharpe \\ Department of Physics, FM-15 \\ University of Washington \\ Seattle, Washington 98195
}

\begin{abstract}
I review the progress made during the last year in lattice calculations of weak and electromagnetic amplitudes.
\end{abstract}

Talk presented at the International Symposium Lattice '89

Capri, Italy, September 18-20, 1989

\section{PREPARED FOR THE U.S. DEPARTMENT OF ENERGY}

This report was prepared as an account of work sponsored by the United States Government. Neither the United States nor the United States Department of Energy, nor any of their employees, nor any of their contractors, subcontractors, or their employees, makes any warranty, express or implied, or assumes any legal liability or responsibility for the product or process disclosed, or represents that its use would not infringe privately-owned rights. By acceptance of this article, the publisher and/or recipient acknowledges the U.S. Government's right to retain a nonexclusive, royalty-free license in and to any copyright covering this paper.

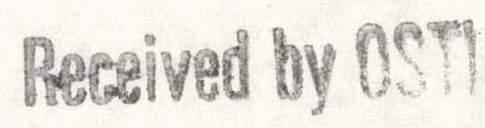

November 1989 


\section{DISCLAIMER}

This report was prepared as an account of work sponsored by an agency of the United States Government. Neither the United States Government nor any agency Thereof, nor any of their employees, makes any warranty, express or implied, or assumes any legal liability or responsibility for the accuracy, completeness, or usefulness of any information, apparatus, product, or process disclosed, or represents that its use would not infringe privately owned rights. Reference herein to any specific commercial product, process, or service by trade name, trademark, manufacturer, or otherwise does not necessarily constitute or imply its endorsement, recommendation, or favoring by the United States Government or any agency thereof. The views and opinions of authors expressed herein do not necessarily state or reflect those of the United States Government or any agency thereof. 


\section{DISCLAIMER}

Portions of this document may be illegible in electronic image products. Images are produced from the best available original document. 


\begin{tabular}{cccc} 
What? & Why? & Who? [6] & Leve] \\
\hline & Nucleon matrix elements & & \\
\hline$f_{\pi} / m_{N}, f_{K} / f_{\pi}$ & check & MANY & 2 \\
Axial vector matrix elements: $g_{A} \ldots$ & check & Sömmer $[7]$ & 2 \\
EM form factors: $G_{M}\left(q^{2}\right), \ldots$ & check & Wilcox, Draper/Liu [8] & 2 \\
Structure functions & check & Rossi $[9]$ & 1 \\
Neutron Electric Dipole Moment & measure $\theta_{Q C D}$ & Goksch [10] & 1 \\
\hline & Heavy-light mesons & & \\
\hline$f_{D}, f_{B}, B_{D}, B_{B}$ & $\bar{D} D$ and $\bar{B} B$ mixing & Eichten, Martinelli [11] & $1-2$ \\
$D \rightarrow K e \nu,(B \rightarrow \pi e \nu), \ldots$ & measure $V_{c s}, V_{u b}$ & El Khadra, $[12]$ Sachrajda $[13]$ & $1-2$ \\
$D \rightarrow K \pi$ & check & Sachrajda, Simone & 1 \\
\hline$B_{K}$ & K decay and mixing amplitudes & \\
\hline & extract $\delta$ from $\epsilon$ & Bernard, Kilcup, [14] Martinelli & 3 \\
$K \rightarrow \pi \pi(\Delta I=1 / 2$ rule $)$ & check & Bernard, Kilcup, Martinelli & 2 \\
$\epsilon^{\prime}$ & over-determine $\delta$ & Kilcup, Bernard & 2 \\
\hline
\end{tabular}

Table 1: Work done on weak matrix elements in the year preceding September 1989

\section{INTRODUCTION and OVERVIEW}

This talk summarizes the work done in the year since September 1988. Most of this work was presented at this conference in the parallel session on weak interactions, and more details can be found in the appropriate contributions. Excellent reviews of the subject as of September 1988 are to be found in the talks at LAT88 [1] by Bernard and Soni, [2] Martinelli [3] and Sachrajda. [4] See also the Ringberg proceedings. [5]

It seems to me that there have been considerable improvements in the calculations during the last year. To discuss these I will divide the calculations into 3 classes: (1) nucleon matrix elements; (2) heavy-light mesons; and (3) kaon decay and mixing amplitudes. Table 1 provides a summary of the progress made, together with the name of the speaker at the parallel session, and a reference, if it exists. The remainder of this article will pick out the highlights from the tablc.

The table also illustrates the importance of the calculations. Roughly half of them will allow us to extract a precise value for an electroweak parameter. To pick a topical example, CLEO and ARGUS have recently reported observations of $b \rightarrow$ uev decays. In principle, this allows an extraction of the Kobayashi-Maskawa (KM) mixing element $V_{u b}$. In practice, this requires calculating an overlap of the initial $B$ meson and the final $\rho$ or $\pi$ wavefunctions. Various phenomenological models yield estimates differing by factors of up to four. The lattice should be able to do considerably better. The ultimate aim is an accurate measurement of all $\mathrm{KM}$ matrix elements, including the $\mathrm{CP}$ violating phase $\delta$. The weak interactions would then no longer be obscured by the messy physics of QCD.

Both the calculations and the experiments have a long way to go before we approach such a rosy situation. As far as the calculations are concerned, however, there is a clear path to follow. The first step is to pin down the results in the quenched approximation. To do this we must figure out how to do the calculations, and then we must control the errors, both statistical and systematic. The systematic errors are due to finite volume, finite lattice 
spacing, the choice of fermion type, and the need to extrapolate to light quark masses. These errors must be reduced to a level at which we can clearly distinguish quenched results from those of full QCD. Of course we don't know the full QCD results for all the quantities, but that is the point of the "checks" listed in Table 1 . We can use these checks to estimate the size of the systematic errors due to quenching. Armed with these estimates, we can then try to do phenomenology using the quenched values of the matrix elements. Finally, when computer power allows, we will do the calculation using full QCD.

There is another systematic error in the calculations of many of the quantities, coming from the need to match lattice and continuum operators. This can be done perturbatively, but only 1-loop calculations exist for nearly all matrix elements. This does not tell us which value of $\alpha_{s}$ to use in the formulae. Given the large ratio of $\Lambda$-parameters, this is a significant uncertainty, which can bc substantially resolved by 2 -loop matching calculations. For most quantities the uncertainties are at the $10 \%$ level, which is not largc enough to bc a worry yet, but eventually the calculations must be done. They can proceed in parallel with improvements in numerical results.

This year has seen the beginning of the appearance of solid quenched values for matrix elements. There are, however, not enough checks to give us a clear idea of the errors of the quenched approximation. My view of the status of various calculations is given in the last column in the table. "Level 1" means an exploratory calculation, mainly setting up the methods, and trying to resolve problems of principle. In some cases, such as the calculations of kaon decay amplitudes, this may well be the most difficult step. The numerical results from this level are to be considered qualitative only. Level 2 means a larger scale numerical calculation, with reasonably small (10-20\%) errors. The results are now quantitative, but the errors are too large to distinguish quenched from full QCD. Level 3 indicates a reliable quenched calculation with small errors. Progress during the last year has resulted in many of the calculations moving up a level. There are now lots of 2's, and a single 3 .

To close the introduction I want to discuss the difference between quenched and full QCD in a particular example: $f_{K} / f_{\pi}$. My aim here is threefold: (A) 1 want to reiterate that having good quenched results is only the first step-much important physics is lost; (B) I want to stress how accurately we must do the quenched calculations to see differences from full QCD; and (C) I want to introduce chiral loops and chiral logarithms, since I will return to them later.

The decay conslants $f_{\pi}$ and $f_{K}$ are the simplest of all weak matrix elements. I am interested here in the quantity $R=f_{K} / f_{\pi}-1$, which is 0.22 experimentally. This is a quantity which is not predicted by chiral symmetry, but rather is a number characterizing QCD. Almost everyone who has ever done a hadron spectrum calculation has, as a byproduct, extracted the decay constants. If we want the errors on $R$ to be, say, one quarter of the experimental value, so that we can definitely distinguish $R$ from zero, we need a $5 \%$ measurement of $f_{K}$ and $f_{\pi}$.

What we really want to do, however, is distinguish $R$ in the full theory from $R$ in the quenched theory. What the quenched theory lacks is loops of fermions. This means that diagrams such as Fig. 1a are missing. Part of these diagrams corresponds to loops of $\pi$, K and $\eta$ mesons, as illustrated in Fig. 1b. It turns out that all the diagrams which give rise to such loops require internal fermion loops, and thus are absent in the quenched approximation. The low momentum part of these loops can be calculated using chiral perturbation theory. 


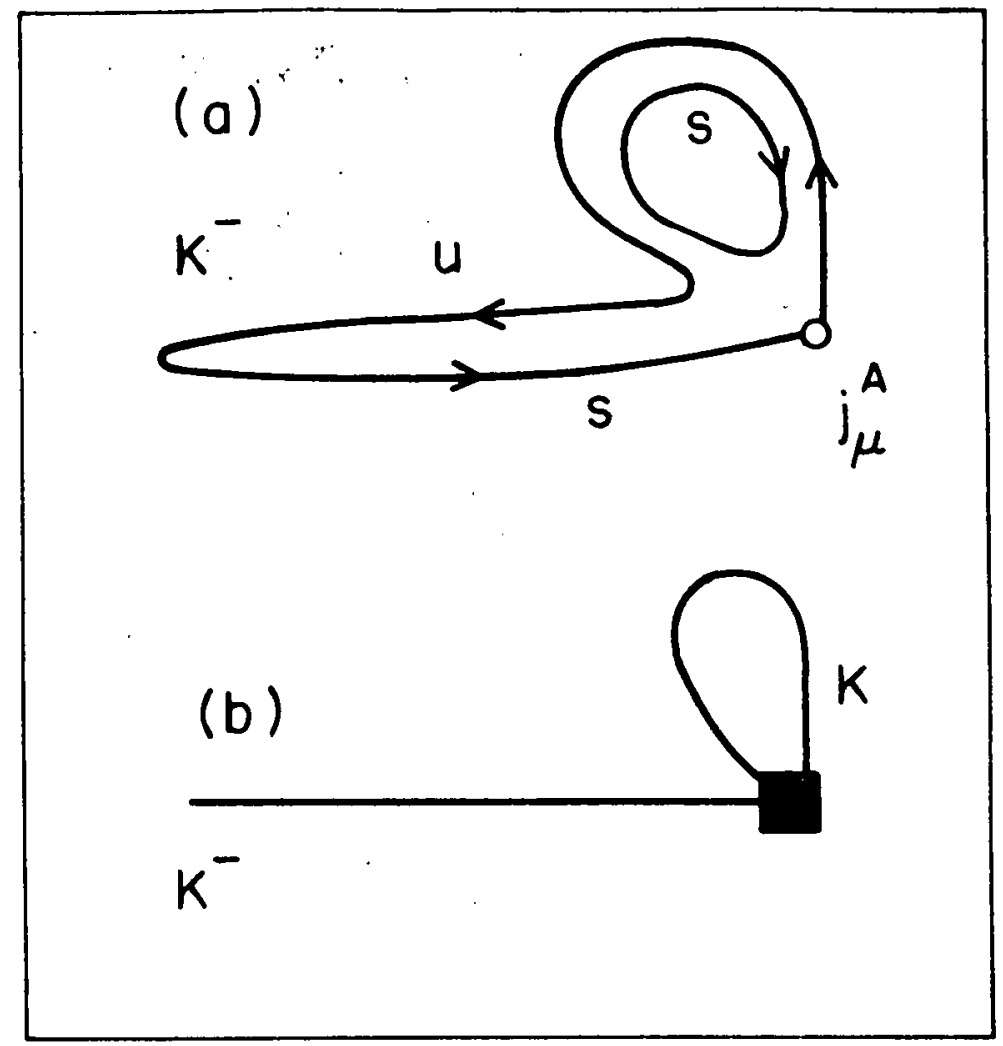

Figure 1: Diagrams for $f_{K}$ absent in quenched QCD.

The resulting chiral logarithm (for $m_{i}=m_{d}=0$ ) is [15]

$$
\left(\frac{f_{K}}{f_{\pi}}-1\right)^{\log }=-0.75 \frac{m_{K}^{2}}{\left(4 \pi f_{\pi}\right)^{2}} \ln \frac{m_{K}^{2}}{\Lambda^{2}}
$$

(with $f_{\pi}=93 \mathrm{MeV}$ ). This is the leading term in an expansion in $m_{K}^{2}$. The scale of the $\log$ is undetermined, but if we take $\Lambda=m_{\rho}$, a scale above which chiral perturbation theory certainly fails, then the log gives a contribution of +0.12 to $R$. I take this as an indication that the quenched approximation may be lacking an effect which contributes roughly half of the experimental value of $R$. If so, then the quenched value would be $R \approx 0.1$. To really distinguish $R=0.1$ from $R=0.22$ we would need to calculate $f_{K}$ and $f_{\pi}$ to $\sim 2 \%$ accuracy. This is considerably below the present errors, which are more like $10 \%$.

The above diagrammatic analysis is called into question by some little noticed work of Morel. [16] He calculated the chiral logs in $\bar{\psi} \psi$ and $m_{\pi}$, in both quenched and full QCD, in the strong coupling $(\beta=0)$ limit using a $1 / d$ expansion. The quenched logs in $f_{\pi}$ can be deduced from this calculation, and they do not vanish, contrary to the claims I made above. This may be an artifact of the limits in which the calculation is being done, or may indicate an error in the diagranunatic approach. However this problem is resolved, it will not affect the qualitative conclusions of the previous paragraph. This is because Morel's quenched logs do differ from the logs in full QCD. 


\section{NUCLEON MATRIX ELEMENTS}

The simplest matrix elements are those of the Hamiltonian itself, i.e. the masses. We would like to compare the quenched values for $m_{\rho} / m_{\text {proton }}, f_{\pi} / m_{\text {proton }}$, etc. to their experimental values. Errors in the masses are at present too large to do this. The extrapolation to physical quark masses is particularly difficult. For a discussion see the talk of Gupta at this conference.

The remainder of this section concerns the structure of nucleons. This is probed experimentally by electroweak currents, i.e. axial and vector currents. Results for axial currents at zero momentum transfer, and for vector currents with momentum transfers up to $\sim(2 \mathrm{GeV})^{2}$ (i.e. electromagnetic form factors), have improved in the last year. From the viewpoint I am adopting here, these calculations serve as important tests of the reliability of the quenched approximation.

\subsection{Axial vector matrix elements}

The Wuppertal group has new results for $g_{A}$ and the $F / D$ ratio. [7] Lel me use $g_{A}$ to illustrate their results. For quark masses such that $m_{\pi} / m_{\rho}=0.63$ and 0.54 they find $g_{A}=1.12(9)$ and 1.10(12) respectively. Recall that experimentally $g_{A}=1.26$. A minimal criterion for a third level quenched calculation is that one should be able to distinguish $g_{A}$ from $g_{V}=1$. Clearly the errors must be reduced substantially. There is also a considerable uncertainty associated with the extrapolation from quark masses $\sim m_{\mathbf{v}} / 2$ to $m_{\mathbf{u}}$ and $m_{d}$.

Because of these errors I judge this calculation to be at level 2. The Wuppertal group has done some sterling work searching out the best smeared operators, and finding other tricks such that they can get reasonable signals for these and other matrix elements. Their calculations are done on small $\left(8^{3} \times 16\right)$ lattices, using an improved fermion propagator. I think it is important to confirm the results on larger lattices with the original action, using the tricks that have been developed.

\subsection{Electromagnetic form factors}

In the last year there has been a steady improvement in the calculations of electric and magnetic form factors of both neutron and proton. The techniques appear to be in place, and the improvements are mainly due to increased computer power. These calculations are now being done at $\beta=5.9-6.0$ on lattices large enough that finite size effects are probably small (e.g. Wilcox [6] uses $16^{3} \times 21$ ). I will illustrate the results using the proton magnetic form factor $G_{M}\left(q^{2}=0\right)$. This is difficult to calculate since one must extrapolate from finite $q^{2}$. Wilcox [6] finds $G_{M}(0) \approx 3.0$, while Ref. 8 quotes $2.3(4)$, where the error is only statistical. These numbers are to be compared to the experimental value 2.79 . There are clearly large systematic errors, so that it is not yet possible to look for any differences between quenched and full QCD.

Wilcox [6] also showed some tantalizing qualitative results. It may be becoming possible to distinguish a dipole from a monopole form in the form factors. There is also some indication for a small negative value of the neutron electric form factor, $G_{E}\left(q^{2}\right)$, at small $q^{2}$, 
i.e. the sign seen by experiment. Unfortunately the errors are too large to draw definitive conclusions on either of these points.

I see no reason why these calculations should not greatly improve given greater statistics. Of course, the usual sources of systematic error will need to be investigated, but there seems to be no obstacle to pinning down the quenched value of the form factors in the next few years.

\subsection{Structure Functions}

The last year has seen only analytical progress. The 1-loop relation between lattice and continuum operators needed for the first moment of the singlet structure functions has been calculated. [9] This is an essential ingredient for accurate calculations.

\subsection{Neutron Electric Dipole Moment (NEDM)}

Aoki and Goksch have recently done a first calculation of the NEDM coming from the $\theta F \tilde{F}$ term in QCD. [10] Naively, one needs calculate the connected correlation function between $F \tilde{F}$ and a neutron, in the presence of a background $\mathrm{E}$ field. This seems like a hopeless calculation-it has proven hard enough to measure the topological charge alone. The trick they use is to do a chiral rotation to put $\theta$ into the mass matrix, and then do a quenched calculation. They find a surprisingly large result, with a behavior which appears to violate chiral perturbation theory. Because of this, it is unclear whether the calculation is theoretically sound. It is clear, however, that this is a subject deserving further study.

\section{$3 \quad$ Heavy-Light mesons}

Heavy-light mesons offer the best possibility for lattice calculations to become an integral part of phenomenology. This is particularly true of $B$ mesons, from which we can learn about the third row and column of the KM matrix.

$B$ meson amplitudes are difficult to calculate because they require that we simulate both the dynamics of the light quarks and gluons, which requires a lattice $\sim 2 \mathrm{fm}$ across, and the dynamics of the $b$ quark, which requires a small lattice spacing $a<1 / m_{b}$. These requirements cannot be met simultaneously by present computers, even in the quenched approximation. We are left with two other options: extrapolating from quarks with $m a<1$, or extrapolating from infinitely heavy quarks. I will discuss the latter option in the section on $f_{B}$. Let me first comment on attempts to cxtrapolate from beluw.

Since present lattices use $\beta=6.0-6.2$, which corresponds roughly to $1 / a=2.0-2.5 \mathrm{GeV}$, it is possible to directly simulate the charm quark. One has $m_{c} a=0.6-0.75$. Although it is clear that lattice artifacts become important when $m a>1$, it is a somewhat contentious issue exactly what is the maximum reasonable value of $m a$ to use. One can address this question by looking at the free quark propagator, and seeing by how much it differs from the continuum propagator. Certainly there are differences proportional to $\exp (-m a)-1$. It is not clcar, however, whal the coefficient of such terms is in the quantities that we wish to compute. Unfortunately, I do not see any way of knowing this without doing calculations 
with a variety of lattice spacings such that $m_{c} a$ varies from 1 down to a value small enough value that we are confident that lattice artifacts are unimportant. Although I think it likely that present values of $m_{c} a$ are small enough so that errors from other sources arc dominant, this really must be checked by pushing calculations to smaller $a$.

It should be clear from this discussion that $I$ view it as dubious to use present results to extrapolate to $B$ mesons. Asymptotic scaling laws provide the only theoretical crutch. For example, $f_{B} \propto m_{b}^{-1 / 2}$ for large $m_{B}$, up to logs. [17] The difficult issue is deciding at what mass such relations begin to hold. All we know at present is that $f_{K}$ is larger $\operatorname{than} f_{\tilde{\pi}}$. Naively I would expect the corrections to the asymptotic form to be suppressed by $\sim m_{\rho} / m_{Q}$ where $m_{Q}$ is the heavy meson mass. If so, asymptotia would begin fairly soon above $m_{D}$. To demonstrate this, however, we need lattice results with small statistical errors from, say, $\beta=6.0-6.3$. The curves for the decay constant as a function of physical $m_{Q}$ must coincide for different $\beta$ and start to follow the asymptotic form. Such results may well be available in the next year or two.

In summary, my attitude is that one cannot yet trust extrapolations to the $\mathrm{B}$ mesons from smaller quark masses. I shall concentrate on the work done directly on charmed mesons. These calculations can teach us two things. First, they allow us to test that the methods work. Second, they can provide direct evidence on the KM element $V_{c s}$. Charmed mesons decays are simpler to calculate than the corresponding kaon decays because, among other things, they are not constrained by chiral symmetry. Thus it is certainly possible that calculations of charmed decay amplitudes may be the most reliable.

A nice feature of the calculations is that finite volume effects will be smaller than for light quark mesons, since heavy-light mesons are smaller. As discissed laler, such effects can be significant in kaon decay amplitudes. The main effect of finite volume is to restrict the allowed spatial momenta.

All calculations to date have been done with Wilson fermions. Chiral symmetry plays no role in these calculations, so there is no pressing need to wheel out the awkward machinery of staggered fermions. Calculations in the last year have been done by the BS collaboration (led by Bernard and Soni, now at Santa Barbara and Brookhaven, formerly known as the UCLA group), by the ELC (European Lattice Collaboration), and by the FNAL group at Fermilab.

Throughout this section I will use the normalization such that $f_{\pi}=135 \mathrm{MeV}$ and $f_{K}=$ $165 \mathrm{McV}$.

\section{1 $f_{D}$ and $B_{n}$}

There have been no new results on $f_{D}$ and $B_{D}$, so I just summarize last year's status. [2] My representation of this is

$$
f_{D} \approx 180 \pm 25(\text { stat }) \pm 30(\text { syst }) \mathrm{MeV}
$$

The systematic error includes the uncertainty in the extrapolation to zero lattice spacing and to physical quark masses, but not that due to quenching. Still, $f_{D}>f_{\pi}$ seems very likely, while the ordering of $f_{D}$ and $f_{K}$ is less clear. $B_{D}$ is found to be within $20 \%$ of unity. The most important direction in which to improve this result is to decrease the lattice spacing, i.e. perform a calculation at $\beta=6.2-6.3$. 


\section{$3.2 f_{B}$}

The alternative to extrapolation from smaller masses is to extrapolate from infinite $m_{b}$, as advocated by Eichten, Lepage and Thacker. [17] In leading order the heavy quark is static, i.e. stays in the same spatial location and simply picks up a phase. This phase consists of the trivial $e^{i m_{b} t}$, which can be extracted by hand, and the path ordered exponential integral of $A_{0}$ in the time direction. The light quark attaches to the ends of this heavy quark propagator:

Only the leading order calculation has been attempted so far. This gives the coefficient of proportionality between $f_{B}$ and $m_{b}^{-1 / 2}$. The big improvement this year has been the introduction of smearing. Eichten [6] finds that smearing the initial heavy quark relative to the light quark is particularly helpful. This improves both the timeslice at which the effective mass reaches a stable value and the number of timeslices for which there is a good signal. The preliminary results presented at this conference are

$$
\begin{aligned}
\frac{f_{B}}{Z} & =410 \pm 100(1.0 \mathrm{GeVa})^{-3 / 2} \mathrm{MeV} \\
\frac{f_{B}}{Z} & =780 \pm 270(1.7 \mathrm{GeVa})^{-3 / 2} \mathrm{MeV}
\end{aligned}
$$

where the first result is for $\beta=5.7, \kappa=.156$, the second from $\beta=5.9, \kappa=.158$. Here $Z$ is a perturbative correction factor, close to unity, to be discussed below. The ELC has had less success with smearing, as explained by Martinelli, [6] but finds results roughly consistent with these.

These results for $f_{B}$ are certainly too large, well outside the range suggested by phenomenological models. The results will improve significantly in the next year - many more values of $\kappa$ and $\beta$, better statistics, better understanding of smearing, etc. There may also be something wrong in the formulation of the calculation. Indeed, discussion of this possibility began at the conference, though no conclusion was reached.

To extract the asymptotic result for $f_{B}$ one must relate the continuum and lattice definitions of the operators which create and destroy the mesons. This calculation is also in a state of flux. The Orsay group [18] have completed the necessary perturbative calculation. Eichten and Hill [19] at Fermilab are doing the calculation using a different method, and their preliminary result disagrees. Numerically this makes no more than a $10 \%$ difference in $f_{B}$, but the theoretical discrepancy needs to be cleared up.

It is extremely important that this method be pursued, since convincing results for $B$ mesons may well require extrapolations from both smaller and larger $b$ quark masses. The method can be easily extended to calculate other quantities, e.g. $B_{B}$ and the semileptonic form factors, and corrections of higher order in $1 / m_{b}$ can also be calculated.

\subsection{Semileptonic form factors}

These are, perhaps, the most important amplitudes to calculate, since they allow a direct measurement of KM matrix elements. The calculations are being pursued by the BS and ELC groups, as discussed here [6] by El-Khadra and Sachrajda, respectively. The program of is to use $K \rightarrow \pi e \nu$ for calibration, $D \rightarrow K e \nu$ to calculate the KM element $V_{c s}$, then to predict $D \rightarrow \pi e \nu$ (the present measurement has very large errors), and finally to extrapolate 
to $B$ decays. $B \rightarrow D e \nu$ will provide another check, while $B \rightarrow \pi e \nu$ and $B \rightarrow \rho e \nu$ will ultimately allow a definitive measurement of $V_{b u}$.

The calculation of these amplitudes is relatively straightforward, because the leptonic part of the calculation can be done perturbatively. What remains is to compute matrix elements of vector currents such as

$$
\left\langle K^{-}\left|\bar{s} \gamma_{\mu} c\right| D^{0}\right\rangle,
$$

for all physical momentum transfers. One must use a source method to compute the hadronic three point function, but this technique is by now standard. The major difficulty is that the need to insert momentum increases the statistical noise. One must also normalize the currents non-perturbatively, [20] which increases the errors. This may be avoided, however, by using the non-local conserved currents. [13] All in all, the calculations are similar in difficulty to those of electromagnetic form factors.

The finite size of the lattice limits the available momentum transfers. This is not, howevcr, a fundamental problem. To extract the KM elements one only needs to compare with the data at a single kinematic point. Of course, it is imporlant to check that the momentum dependence agrees with that found experimentally (which usually means vector or scalar meson dominance), but with relatively few available points I doubt that one can do much more. This is certainly true for $B$ meson decays, for which one can only hope to calculate the amplitude with the final state meson at rest, or almost at rest.

What accuracy would we like in the quenched calculations? The matrix elements are parameterized by two form factors $f_{+}\left(q^{2}\right)$ and $f_{0}\left(q^{2}\right)$, where $q$ is the momentum transfer. Let me concentrate on the former. If the vector current were conserved, i.e. if the quarks in the current were degenerate, then $f_{+}(0)=1$. Deviations come in at second order in the mass splittings, so they should be smaller for the $K \rightarrow \pi$ amplitude than for those involving $D$ mesons. Thus it is really the deviation $f_{+}(U)-1$ which one should try and calculate accurately. Similar comments apply to $f_{v}$. This ceases to be true for heavy enongh $m_{Q}$, since [21] $f_{+}(0) \propto m_{Q}^{-1 / 2}$.

The $K \rightarrow \pi e \nu$ amplitude serves as a test of the methods. The KM element is very well known, and the experimental data is very good. Furthermore, the theoretical understanding of $f_{+, 0}\left(q^{2}\right)$ is already well refined, because one can apply the technology of chiral perturbation theory. [27] Calculations of kaon decay amplitudes are, in general, quite difficult. This one is simpler because of the nearby limit in which the vector current is conserved. This makes the extrapolation in quark masses smooth. The BS group has calculated both form factors with results, illustrated in Fig. 2, which are consistent with the data. This is a satisfactory test of the methods, but a reduction in the errors from $20 \%$ to a few percent is needed before any differences between the full and quenched theories can be seen.

The amplitude for $D \rightarrow K e \nu$ is measured experimentally relatively well. If onc could calculate the form factors from first principles, then one could extract the KM element $V_{c s}$. Of course, within the standard model with three generations, unitarity determines $V_{c s}$ to be very close to 1.0 . It would be nice to check this directly. Let me illustrate the results by quoting $f_{+}(0)$. ELC [13] finds 0.74(17) $(\beta=6.0)$, while BS have 0.88(7) $(\beta=5.7)$. It is too early in the game to worry in detail about the systematic errors. These two numbers are in agreement, and the errors appear good enough to show that $f_{+}(0)$ is definitely smaller than 1 . 
form factor $\mathrm{f}_{0}$ for $\mathrm{K}^{0} \rightarrow \pi^{-} \mathrm{e \nu}$

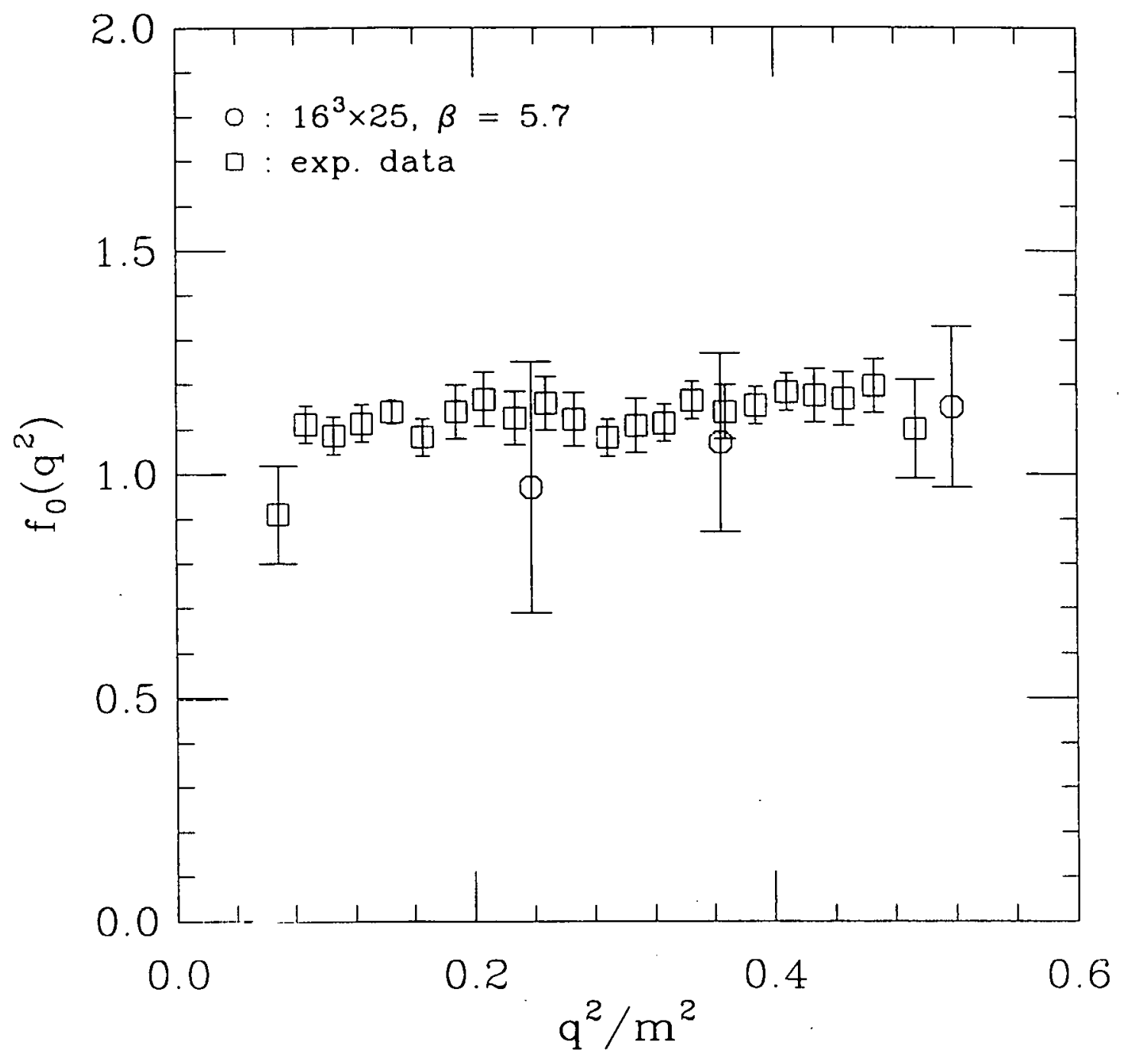

Figure 2: BS data for $f_{0}^{K \pi}\left(q^{2}\right)$ versus $q^{2} / m_{K}^{2}$. 
The numbers are consistent with what is expected if $V_{c s}=1.0$. It should be recalled though, that a variety of phenomenological analyses agree that $f_{+}(0)=0.76-0.77$. [12] These are models, such as the relativized harmonic oscillator, which are tuned to fit other experimental data, and thus are probably quite reliable. It will be hard for lattice calculations to do much better than these models.

As for $D \rightarrow \pi e \nu$, the situation is more interesting. Here we do know the KM element which appears, $V_{c d}$, so the calculation will provide a check on the methods. The phenomenological models disagree significantly, predictions ranging from $f_{+}(0)=0.51$ to 0.69 . Furthermore, experimental data is very scant, so there is the possibility for providing a prediction in advance of measurement. Present lattice errors are $\sim 25 \%$.

In summary, the techniques are in place, and the results for $K$ and $D$ decay form factors should improve substantially in the next year or two. Both BS and ELC groups have larger statistics calculations in progress, which may be goud enough to cstimate the accuracy of quenched calculations of form factors. The extrapulation to the $B$ decay amplitudes can then begin.

\subsection{Nonleptonic decays: $D \rightarrow K \pi$}

In the last year work has begun on calculating non-leptonic $D$ meson decay amplitudes, and is discussed here [6] by Sachrajda (ELC) and Simone (BS). The effort so far has been devoted to the Cabibbo allowed decays $D \rightarrow K \pi$. The experimental data is reasonably good, and the $\mathrm{KM}$ angles are known, so the calculations provide a clean test of the lattice methods.

Fxperimentally there are three decays: $D^{+} \rightarrow \bar{K}^{0} \pi^{+}, D^{0} \rightarrow K^{-} \pi^{+}$, and $D^{0} \rightarrow \bar{K}^{0} \pi^{0}$. These can be written in terms of two reduced amplitudes: the initial isospin is always $I=1 / 2$, the final isospin can be either $1 / 2$ or $3 / 2$. Experimentally one finds that the amplitude to the $I=1 / 2$ final state is about 4 times that to the $I=3 / 2$ final state. This is a lcss dramatic difference than the famous $\Delta I=1 / 2$ rule observed in kaon decays, but deserving ol study nevertlueless.

First let me tell you the good news. The calculations are technically easier than those for kaon decays. The diagrams which contribute are of the type shown in Figs. 3a and 3b: 3a shows an emission diagram; 3b an annihilation diagran. The eye diagram, Fig. 3c, is not present for these $D$ decays, though it does contribute, as the figure shows, to Cabibbo suppressed $D \rightarrow \pi \pi$ decays. It is also present, and very important, in $K$ decays. 'l'he eye diagram is the hardest to calculate, so its absence simplifies the calculation. The momenta of the final state kaon and pion are not small in $D$ decays, so chiral symmetry does not provide any constraints on the amplitude. Thus the extrapolation to small $u$ and $d$ masses is expected to be straightforward.

There is also some bad news. It is not easy to calculate the on-shell amplitude in which the pion and kaon are moving. The problem is to get the correct three-momenta from the limited set available on a finite size lattice. In the pilot calculations that have been done by the ELC and BS groups, the best signals are obtained with all particles at rest. Thus thc weak Hamiltonian is inserting a significant amount of momentum, and one must extrapolate to the physical point. As stressed to me by Soni, it may well be best to compare to the various models which purport to describe $D$ decays at the off-shell point where the lattice calculation is done. The models have been reviewed, for example, by Bigi. [23] One can then 


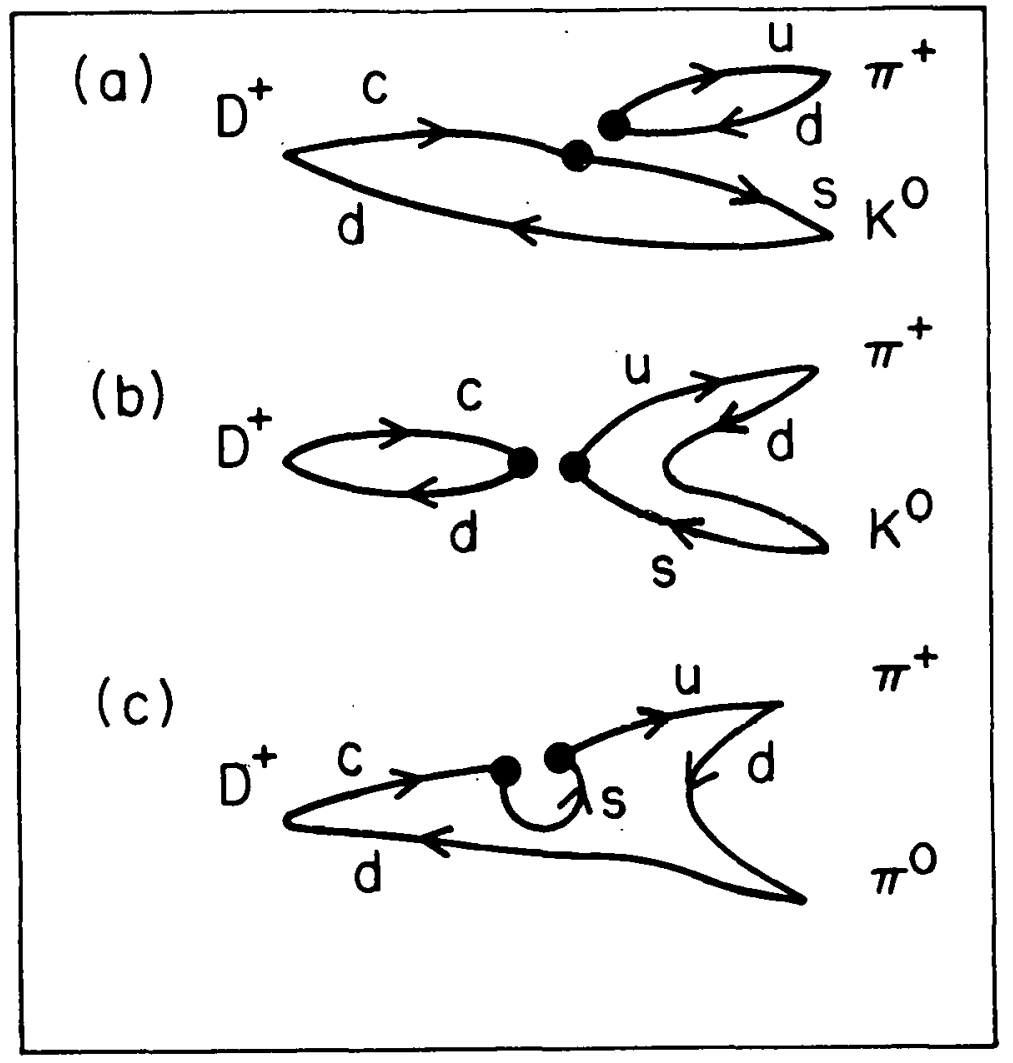

Figure 3: Diagrams contributing to $D$ decays

try and pick the best model, and then use this model to extrapolate to the physical point, and to discuss other decays.

The first studies give mixed results. There is a disagreement between the two groups on how the amplitudes depend on the quark masses. The ELC group finds that the signals decrease as they extrapolate to small light quark masses, eventually disappearing into noise. The BS results show no such decrease. We will have to wait until next year to see whether this disagreement persists.

The BS group has calculated the annihilation diagrams with $m_{s}$ kept different from $m_{u}$. Their results suggest that this diagram (which is ignored in large $N_{c}$ approaches to $D$ decays) can contribute significantly. Thus it is important to use non-degenerate quarks.

I have a personal request concerning the presentation of results. The diagrams of Fig 3a and $3 \mathrm{~b}$ can each be calculated in the factorization approximation, in which gluons joining the two quark loops are left out. No extra propagator calculations are needed to calculate the lattice results in this approximation. Doing so would allow one to see by how much the full amplitude differs from its factorization value. This is a question which the lattice can answer much more easily than giving the absolute values of amplitudes, and it would give a feeling for the size of correlations. Furthermore, it would directly test the first step in some of the phenomenological approaches.

Finally, I wish to raise a point stressed to me by Martinelli. The experimental data shows clearly that there are substantial final state interactions (FSI) in non-leptonic $D$ decays. For example, FSI are responsible for the substantial relative phase between the amplitudes with $I=1 / 2$ and $I:=3 / 2$ final states. FSI also effect the magnitude of the amplitudes. Thus it 
is important that they be handled correctly. This issue is even more crucial for $K$ decays, so I will postpone discussion until the next section.

\section{Kaon decay and mixing amplitudes}

The amplitudes involving only light pseudoscalars are the most difficult to calculate, and have, over the years, received the most attention. This work, much of it theoretical, is now paying off. There are many challenges, however, particularly those posed by strong final state interactions.

These amplitudes are difficult to calculate for a number of reasons. One is that chiral symmetry severely constrains their form. The implementation of chiral symmetry on the lattice is difficult problem, but one which has now been resolved. Because chiral symmetry is important, it makes sense to attack the problem with staggered fermions, which have some chiral symmetry left on the lattice, as well as with Wilson fermions, which completely break the chiral symmetry. My own contributions are as part of the staggered group (STAG), which uses staggered fermions, and is, appropriately enough, staggered across the world (Seattle, Los Alamos, Providence, Bangalore). Our results are presented here by Kilcup. [6] Both the ELC (see Sachrajda [6]) and BS (see EL-Khadra [6]) groups are attacking the problems using Wilson fermions.

In this section I will revert to using the normalization in which $f_{\pi}=93 \mathrm{MeV}$.

\subsection{Kaon B-parameter}

The simplest calculation is of the so-called kaon B-parameter $B_{K}$. This is defined through

$$
\begin{aligned}
& \mathcal{M}=\left\langle K\left|\bar{\sigma} \gamma_{\mu}^{L} d \bar{\delta} \gamma_{\mu}^{L} d\right| K\right\rangle \\
& B_{K}=\mathcal{M} /\left(16 f_{K^{2}}^{2} m_{K^{c}}^{2} / 3\right)
\end{aligned}
$$

Here $L$ represents the insertion of $\left(1+\gamma_{5}\right)$. The definition of $B_{K}$ is illustrated schematically in Fig. 1. The dots represent $\gamma_{\mu}^{L}$, and the averages are over gauge configurations. The denominator is the vacuum saturation approximation to $\mathcal{M}$, which means the factorization approximation multiplied by $4 / 3$. Because $B_{K}$ is a ratio, the uncertainties in $f_{K}$ cancel, making possiblc a much more accurate value for $B_{K}$ than for $f_{K}$.

The CP violating part of $\bar{K} K$ mixing is proportional to $B_{K}$. Since the CP violating part is measured (it is parameterized by $\epsilon$ ), knowledge of $B_{K}$ would allow us to extract a combination of $\delta$ (the phase in the KM matrix), $V_{b u}$ and $m_{t}$. Thus it is extremely important to pin down a value for $B_{K}$.

The simplest guess is $B_{K}=1.0$, i.e. that vacuum saturation approximation is correct. If instead one uses the factorization approximation, then $B_{K}=0.75$. Another estimate usés lowest order chiral perturbation theory (ChPT) to relate $B_{K}$ to the $K^{+}$decay amplitude, finding $B_{K} \sim 0.4$. (This is larger than usually quoted, for reasons discussed in the next subsection.) Thus there is a considerable uncertainty in $B_{K}$, and consequently a need for an accurate lattice determination. It is clear, however, that a quenched lattice determination must be have errors of $5 \%$ or less to be useful. 


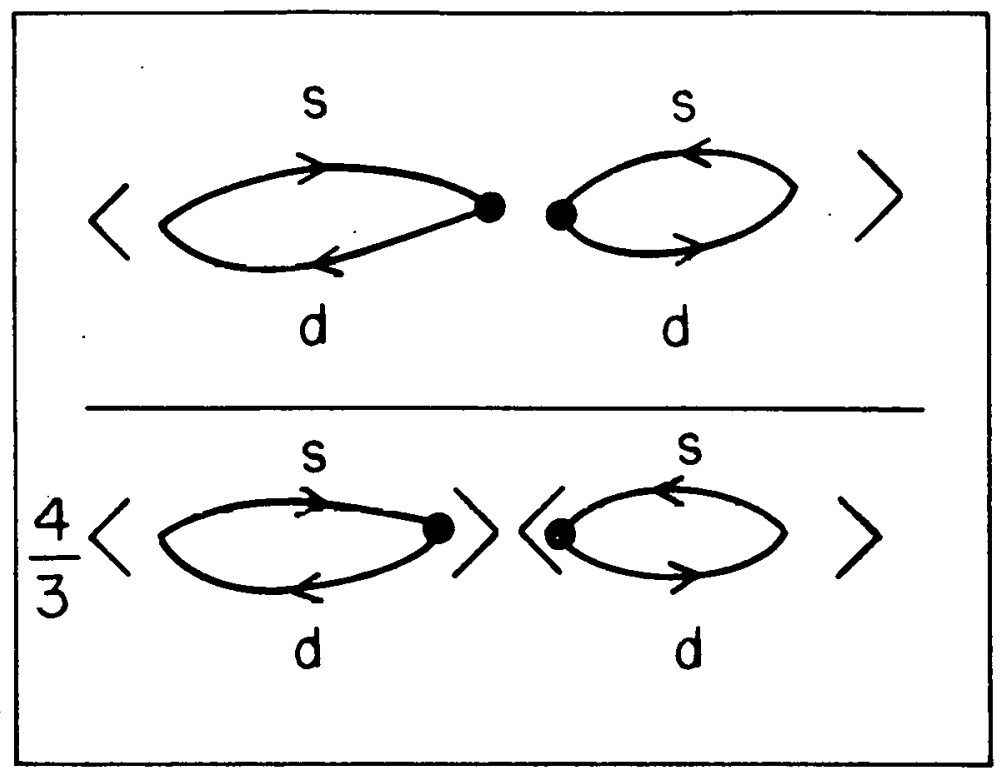

Figure 4: Illustration of the definition of $B_{K}$

$B_{K}$ depends on the scale at which it is calculated: $B_{K}(\mu) \propto \alpha_{s}(\mu)^{2 / 9}$. Vacuum saturation and factorization give scale independent answers. This cannot be correct, so one should treat them only as rough guides. In this talk I will adopt the convention of quoting $B_{K}(2 G e V)$.

Chiral symmetry constrains the matrix element to vanish when $m_{K}^{2} \rightarrow 0$. With Wilson fermions, the LL operator which appears in $\mathcal{M}$ can mix with LR operators. These do not have vanishing matrix elements in the chiral limit. This mixing begins at $O\left(g^{2}\right)$, and the leading perturbative term has been calculated. There is also a correction proportional to $a$ which does not vanish in the chiral limit. Thus, after 1-loop perturbative subtraction, one has (up to terms of $O\left(m_{K}^{4}\right)$ )

$$
\mathcal{M}^{W}=O\left(a, g^{4}\right)+\frac{16}{3} m_{K}^{2} f_{K}^{2}\left(B_{K}+O\left(a, g^{4}\right)\right)
$$

where the $W$ indicates Wilson fermions. Notice that there are corrections to $B_{K}$ of $O(a)$ and $O\left(g^{4}\right)$.

It is not obvious, a priori, which of the $O(a)$ or $O\left(g^{4}\right)$ corrections is the larger. One hint is provided by the range of values that have been found in non-perturbative evaluations of $Z$ factors. For example, $Z_{V}$ varies substantially with the correlator which is used for the calculation, the momentum inserted, and the quark masses. [4] Almost all values differ from the 1-loop perturbative result. This suggests that the major effect is $O(a)$ corrections, which will depend on the method of calculation, rather than $O\left(g^{4}\right)$ corrections, which would be the same for all methods. Nevertheless, for a particular method and quantity, both effects can be important.

The BS group has attempted to remove the higher order perturbative corrections. The idea is to do a non-perturbative subtraction of the LR operators. There are, however, four LR operators, but only two conditions with which to fix their coefficients. Thus there are systematic uncertainties in the subtraction procedure. These are exacerbated by the presence of the $O(a)$ terms, which will depend on which operator is chosen. I am also slightly nervous 
because one of the non-perturbative conditions uses the amplitude for the weak operator to create two kaons, and there may be errors introduced by final state interactions.

In a very interesting development, Rossi [6] explained how, in principle, one could remove the dominant part of the $O(a)$ term. Part of this term is simply the $O(a)$ correction to the mixing with local operators. This can be calculated perturbatively, and subtracted. The residue cannot be removed by adding local operators. The only way to remove this horrible term is to use an improved fermion action. If successful this would replace the $O(a)$ term with one of $O\left(a \times g^{2}\right)$. I am optimistic that this will help considerably, but the work needed to implement this idea is just beginning.

In the meantime, the ELC group quotes a result for $B_{K}$ by fitting $\mathcal{M}^{W}$ to either a linear or a quadratic function of $\left(f_{K} m_{K}\right)^{2}$, and taking the linear piece. The resultant value contains systematic errors of $O(a)$ and $O\left(g^{4}\right)$.

Ultimately, the best approach with Wilson fermions might be to combine the two methods just described, i.e. to remove the $O(a)$ and $O\left(g^{2}\right)$ corrections perturbatively, and then use the non-perturbative subtraction to try and remove the rest.

Because of these complications it may be that staggered fermions are preferable. The advantage of staggered fermions is that Ward Identities imply the absence of constant terms in $\mathcal{M}$, i.e.

$$
\mathcal{M}^{S}=\frac{16}{3} f_{K}^{2} m_{K}^{2}\left(B_{K}+O\left(a, g^{4}\right)\right)+O\left(m_{K}^{4}\right),
$$

where I am assuming that 1-loop perturbative corrections have been included. The corrections to $B_{K}$ are formally of the same order as with Wilson fermions (I see no reason why staggered fermions should have $O\left(a^{2}\right)$ corrections, as is sometimes claimed). Nevertheless, the absence of the constant term removes a source of systematic uncertainty. The price for this is the need to use operators spread out over a $2^{4}$ hypercube. It is also true that the connection to matrix elements in QCD is more involved than with Wilson fermions, since one has four timcs the number of continuum fermions. I do not think this is a problem of principle. [24] It does mean, however, that the two loop perturbative calculations, which have to be done eventually, will be very hard.

I give the results for $B_{K}(2 \mathrm{GeV})$ from the three groups in table 2 . The first error is statistical, the second an estimate of the systematic error due to subtracting LR operators. I have noted the number of configurations and the spatial lattice size under each result. All the BS and STAG results, as well as those from ELC at $\beta=5.7$, are new this year. I have quoted the ELC results from their linear fits, using the difference between linear and quadratic fits as an estimate of the systematic error. The DS results are from lincar fite, with their quoted systematic error. For staggered fermions no fils ale needed.

I have not included any error due to the use of only 1-loop perturbation theory. This error remains to be calculated. I do not think, however, that it will be a very large effect, since the perturbative corrections to $B_{K}$ are quite small. For staggered fermions they are $\sim 1 \%$, so even a $100 \%$ uncertainty in these corrections is only a small effect.

The US Department of Energy Grand Challenge grant of 8000 Cray-2 hours during the last year to the joint BS-STAG group has been responsible for a dramatic improvement in their results. The results at $\beta=5.7$ and 6.0 from these two groups are based on the same ensemble, with the UCLA group using alternate lattices. At $\beta=6.0$, the two lattices $\left(16^{3}\right.$ and $24^{3}$ ) allow a test of finite volume effects. The three values of $\beta$ allow a test of scaling. 


\begin{tabular}{clll}
$\beta$ & ELC $[25]$ & BS & STAG $[14]$ \\
\hline 5.7 & $.86(10)(25)$ & $.96(10)(11)$ & $.94(3)$ \\
& {$[110 @ 10 \times 12 \times 16]$} & {$\left[32 @ 16^{3}\right]$} & {$\left[30 @ 16^{3}\right]$} \\
\hline 6.0 & $.81(16)(6)$ & $.83(11)(11)$ & $.70(2)$ \\
& {$\left[30 @ 10^{2} \times 20\right]$} & {$\left[19 @ 16^{3}\right]$} & {$\left[31 @ 16^{3}\right]$} \\
& & $.66(8)(4)$ & $.70(1)$ \\
& & {$\left[8 @ 24^{3}\right]$} & {$\left[15 @ 24^{3}\right]$} \\
\hline 6.2 & $.89(20)(28)$ & & $.70(3)$ \\
& {$\left[15 @ 16^{3}\right]$} & & {$\left[27 @ 18^{3}\right]$} \\
\hline
\end{tabular}

Table 2: Results for $B_{K}(2 \mathrm{GeV})$

We will see more of these lattices later.

The ELC results at $\beta=5.7$ are from a very high statistics run on APE designed to reduce the statistical errors in various quantities.

All results for $\beta \geq 6.0$ are consistent. This is very important, since the staggered and Wilson fermion calculations are very different. The staggered fermion results are better, both because a subtraction is not needed, and because of the smaller statistical errors. I think the latter is due to the methods employed, rather than a feature of staggered fermions. The use of wall sources allows us to sum the amplitude over all spatial positions of the weak operator, giving a large increase in statistics. This trick is due to Greg Kilcup. Similar methods can be used for Wilson fermions, and perhaps should be tried.

The staggered data is good enough to see a clear deviation between $\beta=5.7$ and 6.0. This confirms what was known from earlier studies, namely that we need to use $\beta \geq 6.0$ to approach the continuum limit. For $B_{K}$ the data is consistent with having reached this limit, though a calculation on a larger lattice at $\beta=6.2$ is needed to confirm this. This will be attempted next year. The two lattice volumes at $\beta=6.0$ give results in complete accord, and the $24^{3}$ lattice is large enough to convince me that finite volume effects are small. The STAG group has also checked that the extrapolation from a kaon made up of two quarks with masses $m_{s} / 2$ to the physical kaon will change the result by less than the statistiral error. Decause of all this, I have taken the bold step of labeling calculations of $B_{K}$ as having reached level 3 . This means that I think we know the quenched number to sufficient accuracy that we can (almost) stop doing the calculations and move onto other things.

Of course, what we really want is the value in full QCD. To use the quenched answer, we need an estimate of the error due to quenching. This is one purpose of calculating $K$ decay amplitudes, as described below. Unfortunately, without such an estimate, the accurate quenched answers are of little phenomenological use. Nevertheless, I think we can be pleased that we have got a first firm foothold.

\subsection{Finite volume effects in $B_{K}$}

I now wish to briefly indulge myself, and describe some interesting results that the STAG group has found, which give me some confidence that we know what we are doing. [14]

One effect of using the quenched approximation is to remove or alter chiral logarithms, as discussed above for $f_{K} / f_{\pi}$. It turns out, however, that the quenched $B_{K}$ has, for degenerate 


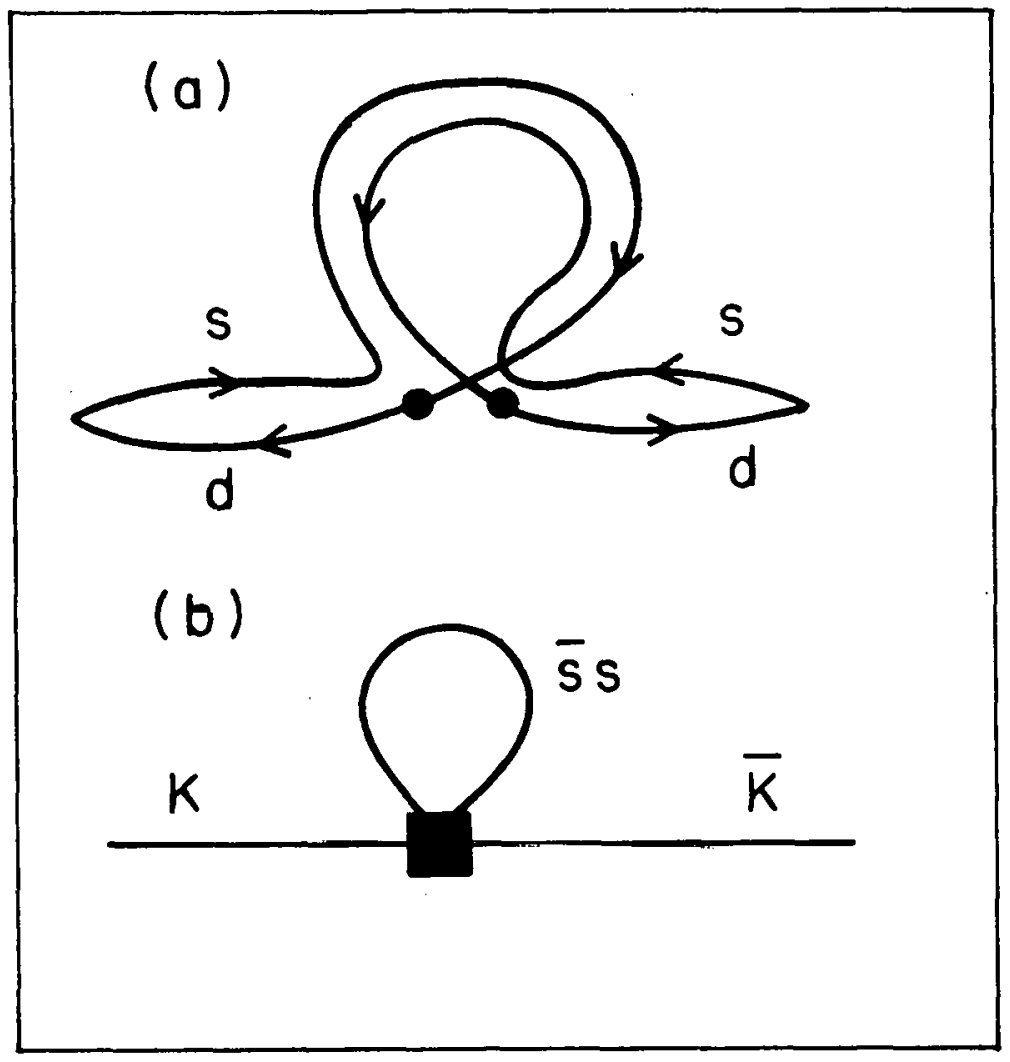

Figure 5: Diagrams leading to chiral logs in $B_{K}$

quarks, the same chiral logs as in full QCD. [26] These arc terms $\propto m_{K}^{2} \log m_{K}^{2}$ which vanish in the chiral limit. The quark diagrams which give rise to these logs are exemplified by Fig. $5 \mathrm{a}$, and clearly do not require internal fermion loops. The corrcsponding meson diagram is shown in Fig. 5b, and it is straightforward to calculate the coefficient of the logs. I should stress that the chiral logs in $\mathcal{M}$ alone are very different in the quenched and full theorics, but these differences are canceled when dividing by $f_{K}^{2}$ (see Eq. 6). The fact that the logs are the same as in the continuum suggests that the effect of quenching on $B_{K}$ may be less than that on $f_{K}$. This is not a rigorous statement because differences in non-logarithmic terms are unknown. Another caveat is that, as discussed in the introduction, the work of Morel suggests that the diagrammatic analysis which I have used may be incomplete.

For my purposes here, the most important consequence of the chiral logs is that they lead to a predictable finite volume dependence. Physically, this arises as follows. The chiral logs come from the long distance part of loops, such as that in Fig 5b, in which the meson starts and ends at the same point. Now, with periodic boundary conditions, the mesun can travel from a point to its image in the adjacent periodic cell. This gives rise to the leading order finite volume effect which is suppressed by $\exp \left(-m_{K} L_{s}\right)$, with $L_{s}$ the spatial lattice size. This can be calculated following the methods of Gasser and Leutwyler. [22] It turns out that $B_{K}$ is predicted to differ by less than a percent between our two lattice sizes at $\beta=6.0$ (see table 2). This is smaller than our statistical errors.

This is a consistency check, but proves little. To do better, we use one of the many other matrix elements which we calculate at an intermediate stage. This is the vector-vector part of the LL operator, with the contractions of the external quarks constrained. [24] We can 
divide out by $f_{K}^{2} m_{K}^{2}$ and form another B-parameter: $B_{V}$. It turns out that the chiral logs for this operator are not suppressed by $m_{K}^{2}$, i.e. $B_{V} \propto \log m_{K}^{2}$, so that they dominate the matrix element at small enough $m_{K}^{2}$. Consequently, the associated finite volume effects are predicted to be much larger. The result is $[26]\left(y=m_{K} L_{s}\right)$

$$
B_{V}=c_{V}\left(\ln \frac{m_{K}^{2}}{\Lambda^{2}}-6 e^{-y} \sqrt{\frac{2 \pi}{y}}+O(1)\right) .
$$

For our lattices $y$ ranges from 3.8 to 9.7. The constant $c_{V}$ can only be estimated to within a factor of $\sim 2$.

Our $\beta=6.0$ data for $B_{V}$ is shown in Fig. 6. The finite volume effect is clear, and is consistent in sign, shape and magnitude with Eq. 10, as is shown by the fits in the figure. Note that $B_{V}$ diverges as expected, but it turns out that the fit requires constants and higher order terms, and is not very impressive. What I am peddling here is the finite volume effect associated with the logarithm. We expect it, and find it, and this is only possible given our small statistical errors. This gives me confidence that the lack of a finite volume effect in $B_{K}$ itself is reasonable.

\section{3 $K \rightarrow \pi \pi$ decay amplitudes}

The CP conserving part of the kaon decay amplitudes serve as a crucial testing ground for calculations. In the standard model, we have all the ingredients needed to calculate the decay amplitudes. The measured rates show a spectacular hierarchy. This was codified by Gell-Mann, 35 years ago, as the $\Delta I=1 / 2$ rule: $A_{0} / A_{2} \approx 22$. Here the amplitudes are $A_{0,2}=A(K \rightarrow \pi \pi(I=0,2))$. Progress has been made in the last year towards understanding this rule.

The calculations are difficult because of the constraints of chiral symmetry, because of mixing with lower dimension operators, and because there are two particles in the final state. Much work has led to two different methods of calculation. In the first, $A(K \rightarrow \pi \pi)$ is calculated with all particles at rest, and all quarks degenerate. All particles are on-shell, so this is a well defined amplitude, but the weak Hamiltonian must insert momentum. I will refer to this amplitude as $A^{\text {rest }}$. Lowest order ChPT is used to relate $A^{\text {rest }}$ to the physical. amplitude, $A^{\text {expt }}$. This method is the cleanest theoretically for Wilson fermions, and involves a relatively small chiral extrapolation. The major disadvantage is that the results can be obscured by final state interactions (FSI). I will argue shortly that this may be a very serious disadvantage. This method has been used, with Wilson fermions, by BS and ELC groups.

The second method of calculating $A_{0,2}$ is to evaluate the $\mathrm{K}$ to $\pi$ transition amplitude $A^{K \pi}$. One then uses lowest order ChPT to relate this to the physical amplitude. This method avoids FSI, which is both its major advantage and disadvantage. The advantage is that the calculations become tractable. Even the notorious eye-diagrams have begun to yield results this year. The disadvantage is that ChPT is expected to be much less reliable than for the first method. This is mainly due to FSI, which, though they may be numerically large, are formally of second order in ChPT. One must eventually deal with the problems of FSI, but it may be prudent, for the moment, to proceed in stages. Staggered fermions, and the STAG group, enter the game once we consider only $K \rightarrow \pi$ amplitudes; in fact, 


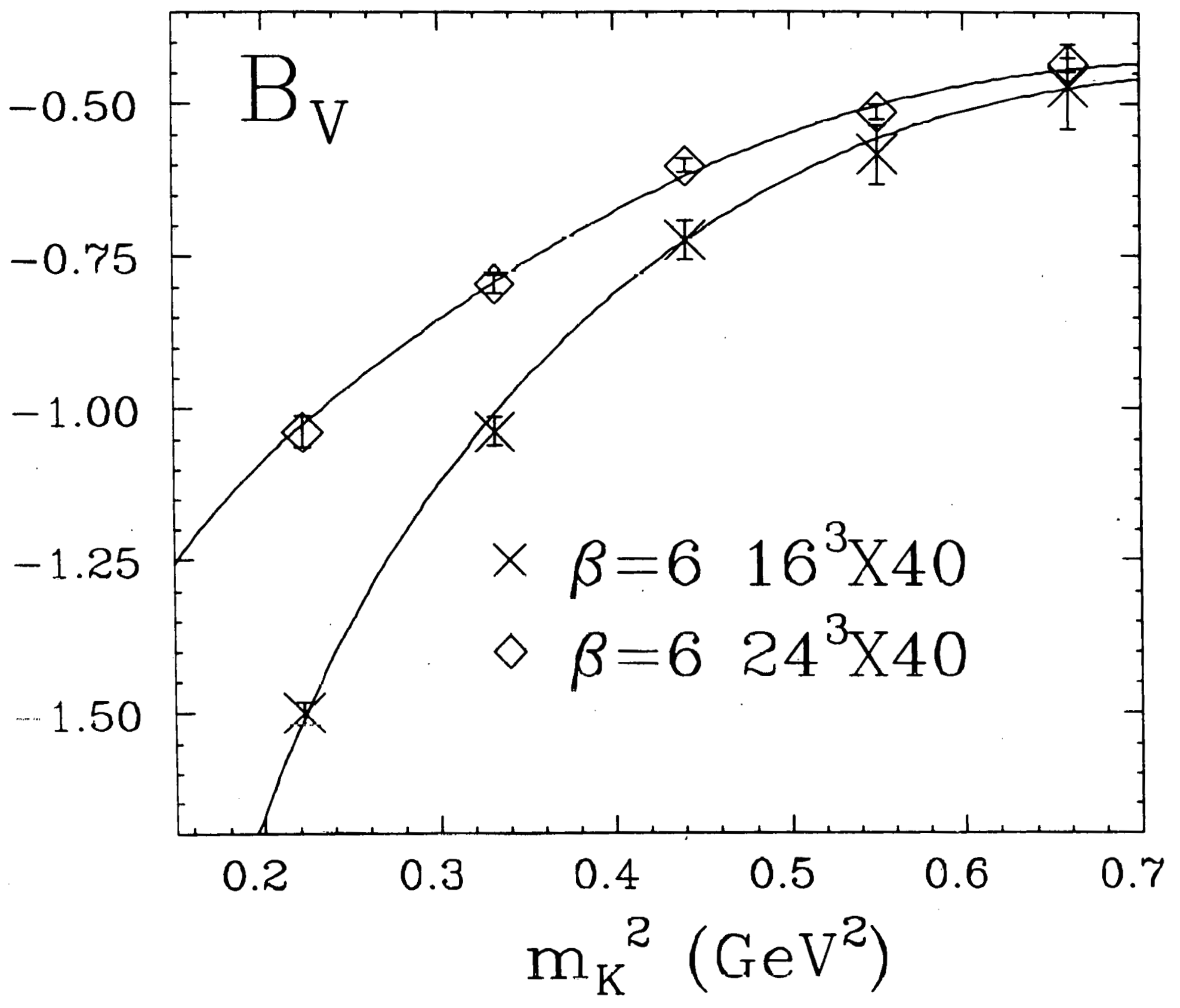

Figure 6: Staggered results for $B_{V}$ 
the calculation is simpler for staggered fermions. The ELC group has also used this method with Wilson fermions.

\subsubsection{Final State Interactions}

Two developments in the last year make it clear that FSI are very important in $K$ decays. First, both the BS and ELC groups have seen evidence for large FSI in $A_{0}^{\text {rest }}$. Second, a paper from the Toronto group has appeared, [28] which claims that FSI make a large contribution to the $\Delta I=1 / 2$ rule. Their observation is that, since FSI are attractive for $I=0$ pions (the phase shift is positive), the outgoing pion wavefunctions will be distorted so as to have more overlap with the initial kaon, thus increasing $A_{0}$. Conversely, the repulsive $I=2$ pion interactions will decrease $A_{2}$. As a qualitative statement this is clearly correct. What came as a big surprise to me, however, is the size of these effects. They find that $A_{0}$ is enhanced by $1.5-2.0$, while $A_{2}$ is reduced by a factor of $\sim 2$. These are somewhat model dependent numbers, with the result for $A_{2}$ being most reliable. I strongly recommend reading their paper: if it is correct it is very important; if wrong, someone should find the error. My opinion is that there is indeed a large effect, though I would not be surprised if the depression of $A_{2}$ were by a factor of 1.5 rather than 2.0 .

If this paper is correct, then as much as a factor of 4 in $A_{0} / A_{2}$ can come from FSI. I will discuss the numbers in more detail below.

These developments make it imperative to include FSI properly in lattice calculations. To do this, one must use final states which are eigenstates of the QCD Hamiltonian, which here means isospin eigenstates. FSI change the energy of the two pion final state from $2 m_{\pi}$ to $2 m_{\pi}+\Delta$. For large volumes, $\Delta \propto 1 / L_{s}^{3}$, with the proportionality constant related to the phase shift. The detailed form is given by Luscher. [29] 'To get the correct answer for the amplitude, one must use the correct exponential fall-off, i.e. $\exp \left(-t\left(2 m_{\pi}+\Delta\right)\right)$. Even if $\Delta$ is small, $t$ can become quite large, so omission of the FSI energy shift can be significant. Finally, of course, one must send $L_{s} \rightarrow \infty$, and check that the resulting amplitude tends to a finite limit. This limit will include the effect of distorted pion wavefunctions. The answer will be real, however, since the calculation is in Euclidean space. The phase of the Minkowski amplitude has to be calculated indirectly using the value of the finite volume energy shift, $\Delta$.

This all means that to calculate, for example, the amplitude for $K^{0} \rightarrow \pi^{+} \pi^{-}$, which is a linear combination of $A_{0}$ and $A_{2}$, one must first calculate the magnitudes and phases of $A_{0}$ and $A_{2}$ as outlined above, and only then combine them.

$I=2$ final state interactions come from gluon exchange and from (anti-)quark interchange, shown respectively in Figures $7 \mathrm{a}$ and $7 \mathrm{~b}$. The former is higher order in ChPT, and is likely to be small for physical pions. Both these diagrams are present in the quenched approximation, so that one can hope to study $I=2$ FSI. Such studies are being initiated by a. number of groups.

For $I=0$ pions, one also has the annihilation diagrams of Fig. 7c. In full QCD, one can iterate this diagram, while the interaction only occurs once in the quenched approximation. It will thus be much harder to study $I=0 \mathrm{FSI}$. It is already clear, however, that these FSl are important. For example, when calculating $A_{0}^{\text {resl }}$ in the quenched approximation, the kaon can decay into an off-shell scalar particle (the $\sigma$ ), which in turn decays to a pion pair. 


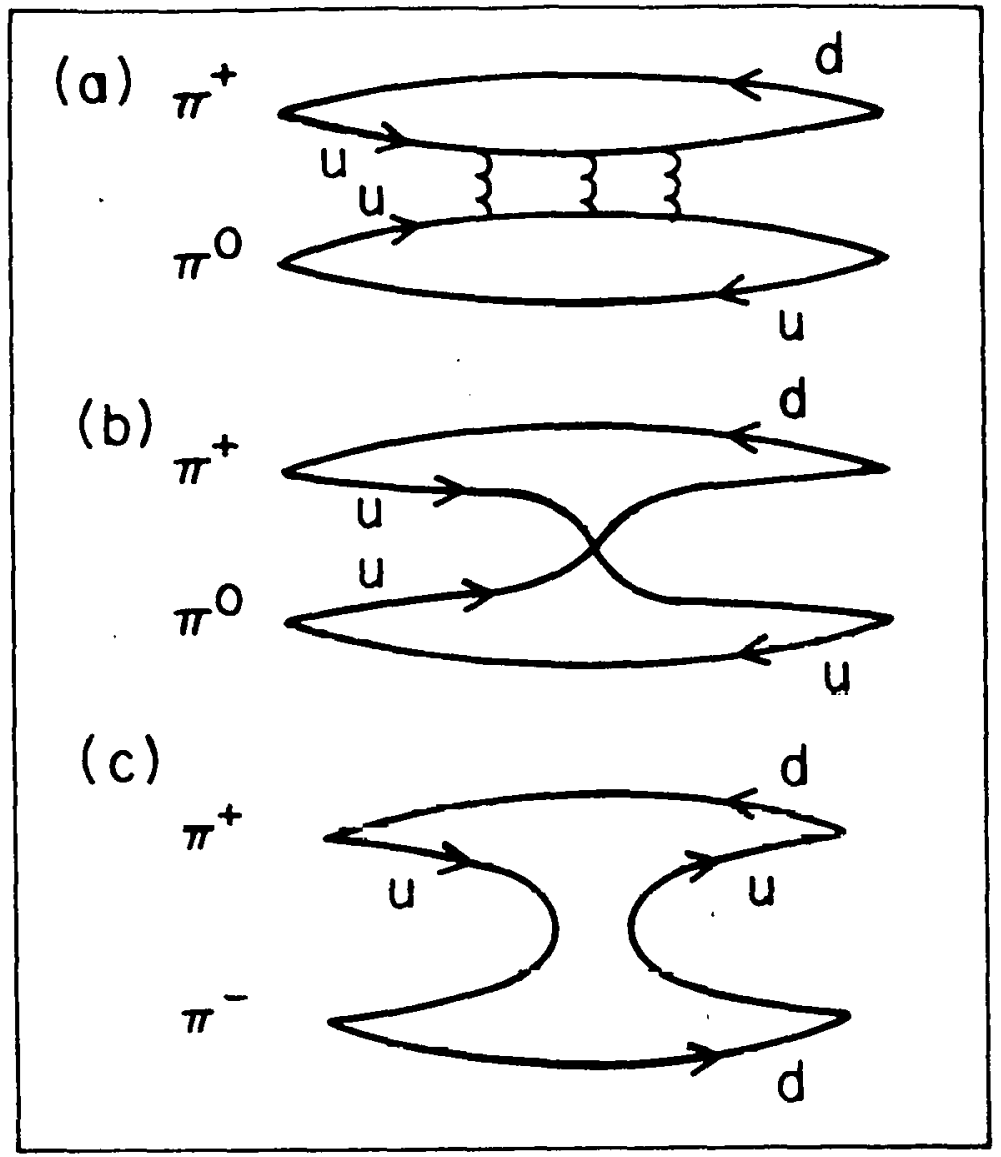

Figure 7: Two pion final state interactions

This effect completely obscures the results for $A_{0}^{\text {rest }}$, in a way which is not understood.

Luscher has a graph showing the energy shift expected for a physical two pion state in a finite box. [29] The fractional increase in the energy of two piuns in an $1-2$ statc in a box $2.4 \mathrm{frn}$ acruss is about 10\%. Thio is the largest volume used to datie. For $l=0$ pions, the fractional reduction in energy is about three times larger. Of course, lattice pions are heavier, which will probably reduce the shifts, but these numbers illustrate how large the effect can be on present lattices.

\subsubsection{Results for $A_{2}$}

The $A^{K \pi}$ method for $A_{2}$ involves calculating the same matrix element as for $B_{K}$. The result $B_{f_{i}}\left(2\right.$ FeV $\left._{\mathrm{F}}\right)=0.7$ converts into $A_{2}=1.75 A_{2}^{\text {expt }}$.

Another way of saying this is that lowest order ChPT converts the physical value $A_{2}^{\text {vit?t }}$ into a prediction that $B_{K}(2 \mathrm{GeV})=0.4$. This is larger than the value usually quoted [30] by a factor of $f_{K}^{2} / f_{\pi}^{2}=1.5$. This difference is a higher order effect, but I think the choice I have made is well motivated. In either case, there is a large discrepancy with experiment, much larger than the errors, which are of a few percent.

A similar discrepancy is apparent in the calculations which use $A_{2}^{\text {rest }}$. The results from these calculations are shown in Fig. 8 as a ratio to the physical value. I have only shown results with relatively small errors and with $\beta \geq 6.0$. The BS results are from the Grand Challenge calculation. The ELC results are old, [25] and come from a smaller lattice. The ELC group also has a new result from their high statistics calculation at $\beta=5.7$. I do not 
have the numbers to allow me to add it to the graph, but their result can be expressed as $A_{2}=2.0(2) A_{2}^{e x p t}$. The results do not yet have small enough errors to try to differentiate between different values of $\beta$.

Superficially, both methods of calculation appear to agree that $A_{2} \sim 2 A_{2}^{\text {expt }}$. This is the conclusion drawn by the ELC group. In this view, the quenched result for $A_{2}$ is fairly well known, and appears to be in substantial disagreement with experiment. This disagreement would be due to two effects. First, the use of the quenched approximation, and, second, an incorrect extraction of the value of $A_{2}^{\text {expt }}$ from the data. Such an error is possible because isospin is broken by electromagnetism, and by the non-vanishing value of $m_{u}-m_{t}$. This can feed parts of $A_{n}$ into $A_{2}$. The calculation of this feed-down is very difficult, as discussed by Bernard and Soni [2] and emphasized by the ELC group. Factors of two effects may be possible, although explicit calculations of some contributions, such as $\eta-\pi^{0}$ mixing, find them to be at the $10-20 \%$ level. This is a very unsatisfactory situation, one that clearly needs more work.

A year ago I would have accepted this resolution. However the Toronto group's paper on FSI provides an alternate explanation. The calculations of $A^{K \pi}$ do not include the FSI depression of $A_{2}$. The required factor of 1.75 is precisely of the magnitude expected. As for the results for $A_{2}^{\text {rest }}$, they are suspect for the following reasons. First, the extraction of $A_{2}^{\text {rest }}$ is being done wrongly, because the FSI energy shift $\Delta$ is not being included. One can test for this for by looking carefully at the exponential fall-offs, and by using different pion source times. Second, if FSI are large, then there will be a large correction to lowest order ChPT, which will show up as a slope in the curves in Fig. 8. I do not know which sign the slope should have. Third, if the FSI mass shift has not been included, the results should depend on the spatial volume.

The BS results in Fig. 8 do show some indication of the second and third features, but the statistics are not yet good enough to be sure. The ELC results at $\beta=5.7$, however, show no evidence for any FSI effects.

The above speculations are meant to encourage further study of FSI, and of $A_{2}^{\text {rest }}$. Present results cannot yet discriminate between the two scenarios I have just outlined. We should, however, be able to settle this question in the next couple of years. Of course, the final conclusion may well involve a combination of the options.

\subsubsection{Results for $A_{0}$ and the $\Delta I=1 / 2$ rule}

The $\Delta I=1 / 2$ rule is really two problems: how to get $A_{2}$ small enough, and how to make $A_{0}$ large enough. We have seen how we must come up with an extra factor of $\sim 1.75$ reduction to solve the first problem. In this section I shall assume that this factor has been found. I shall also assume that FSI provide a factor of 1.5-2 enhancement in $A_{0}$. Then, if we use amplitudes calculated in $K \rightarrow \pi$ matrix elements, which is all that can be done for $A_{0}$ at present, we have to aim for the ratio

$$
R=\frac{A_{0}}{A_{2}}=\frac{22.2}{(1.5-2) \times 1.75}=(6.3-8.5),
$$

rather than 22.2 . 


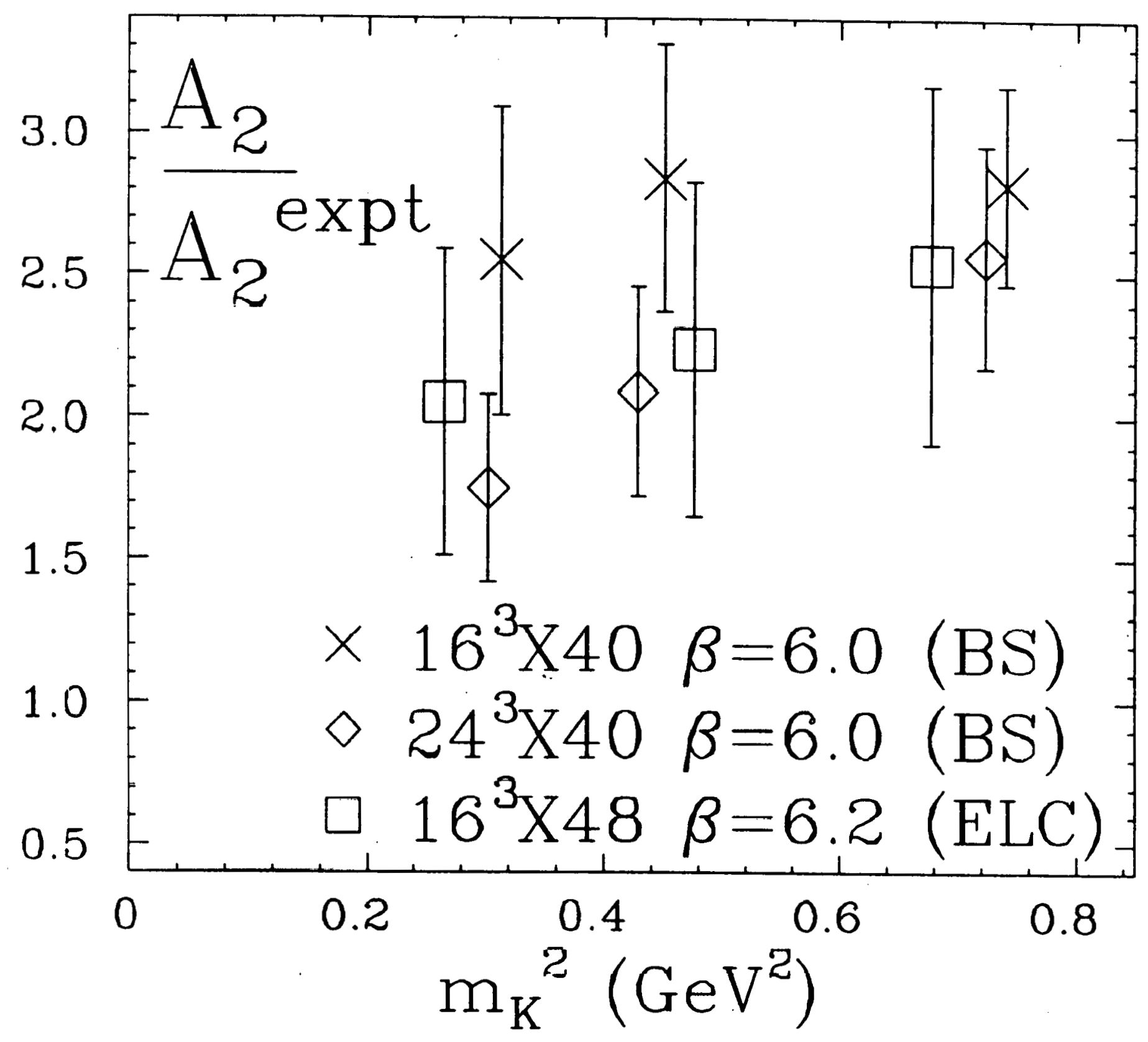

Figure 8: Results for $A_{2} / A_{2}^{\text {expt }}$ deduced from $A_{2}^{\text {rest }}$ 
It is amusing to see how the various contributions to $R$ accumulate. If we begin at scale $\mu=m_{W}$, and use the factorization approximation, then $R=\sqrt{2}$ due to a Clebsch. Running down to $\mu \sim 2 \mathrm{GeV}$, and including QCD corrections (I am using $c_{+}=0.8$ and $c_{-}=1.56$ in a normalization in which both start at 1.0), but still using factorization, gives another factor of 1.7. Using the lattice values for the eight-diagram matrix elements, instead of factorization, gives another factor of 1.5 . This factor is known with $5 \%$ statistical errors for staggered fermions, but this is a preliminary result which has not been fully checked out. Wilson fermions (both BS and ELC) agree, with somewhat larger errors. Altogether these effects multiply (for staggered fermions) to $R=3.6(2)$. So the eight diagrams take us half way towards our lowered goal.

This leaves the eye diagrams. The STAG group finds the results for eights +eyes (again preliminary) shown in Fig. 9. At the physical kaon mass, the result is not impressive. At higher masses, though, the results definitely indicate that eye diagrams go in the right direction, and might have the required magnitude. The ELC group has a tantalizing result from their run at $\beta=5.7$ on APE. They find (from eights+eyes) $R=12 \pm 6$ from 75 configurations, but $R=12 \pm 12$ from 110 configurations. This is a preliminary result, and they are checking for errors in their configurations.

The situation is very interesting. It seems to me that the makings of an understanding of the $\Delta I=1 / 2$ rule have appeared. The rule follows from an accumulation of factors of $1.5-2.0$. On the other hand, various of these contributions are either not well known, or need confirmation: eye diagrams and FSI in particular. The results for the eyes should improve substantially in the next year, while studies of FSI are just beginning. Thus, while there has been progress, we remain a long way from using $K$ decay amplitudes to calibrate the quenched approximation!

There are striking similarities between the scenario just outlined and the large $N_{c}$ explanation of the $\Delta I=1 / 2$ rule. [31] It would be worth understanding these better. Another interesting question is how the above discussion extends to the $\Delta I=1 / 2$ rule in non-leptonic hyperon decays.

\section{$4.4 \epsilon^{\prime}$}

The STAG group has new results, presented here by Kilcup [6] for the matrix element of the operator which gives the largest contribution to $\epsilon^{\prime}$. Our preliminary result, from the $16^{3}$ lattices at $\beta=6.0$, is $B_{6}=.87(9)$. This means that vacuum saturation approximation is working reasonably, for it would imply that $B_{6}=1$. Previously we had found a smaller value, [32] $B_{6}=.4(3)$. Neither result has perturbative corrections included, and these corrections will be different since different operators were used in the two calculations. The new result comes from a large lattice with smaller spacing, and is therefore to be preferred. A complete analysis is in progress, including results on the $24^{3}$ lattice.

The BS group is calculating the same matrix element with Wilson fermions using the $K \rightarrow \pi \pi$ method. At present they have no results, but hope to have some by next year. 


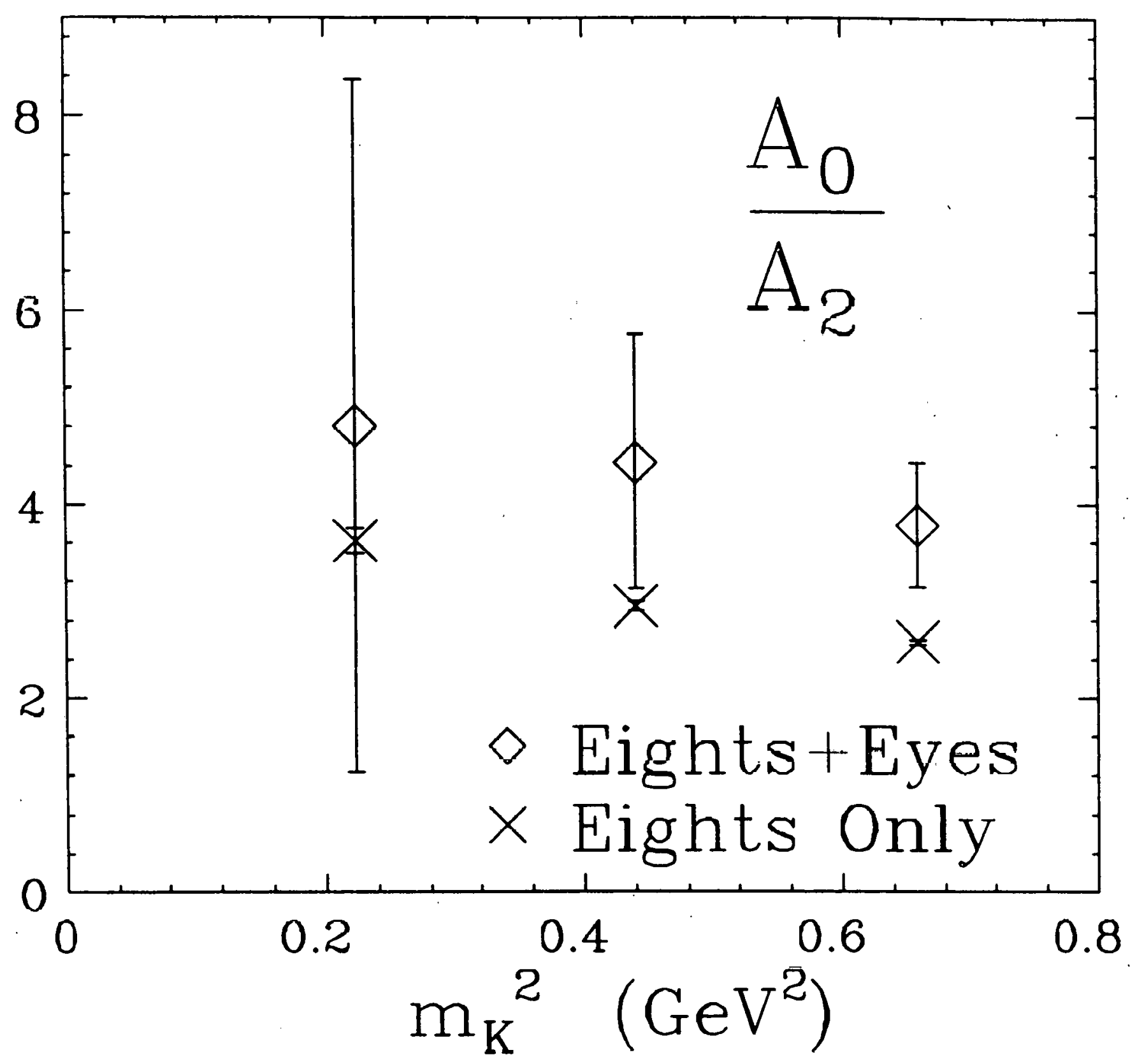

Figure 9: Staggered fermion results for $A_{0} / A_{2}$ 


\section{$5 \quad$ Future directions}

I will simply list what I think are interesting avenues of research, many of which havc already begun.

- Final state interactions

- Removing $O(a)$ terms for Wilson fermions using an improved action

- Understanding $O(a)$ splittings amongst the 16 pions for staggered fermions

- Understanding chiral logs in the quenched approximation

- Understanding the effect of loops of the $\eta^{\prime}$ in the quenched approximation. This particle gives rise to loops which do not follow the standard ChPT power counting rules

- Extrapolating from the quenched to full QCD by calculating derivatives with respect to $n_{f}$

\section{ACKNOWLEDGMENTS}

Particular thanks go to Claude Bernard and Greg Kilcup for many discussions over the last year. In preparing the talk I had useful conversations with S. Aoki, E. Eichten, A. El-Khadra, A. Gocksch, R. Gupta, B. Hill, G. Martinelli, A. Patel, C. Sachrajda, J. Simone, A. Soni, and W. Wilcox. I thank the organizers and the secretariat for their efforts, and for showing me a little slice of Neapolitan culture. This work was supported in part by the Department of Energy Outstanding Junior Investigator program through contract DE-AT06-S8ER40423.

\section{References}

[1] Nucl. Phys. B (Proc. Suppl.) 9 (1989)

[2] C. Bernard and A. Soni, Ref. 1, p155

[3] G. Martinelli, Ref. 1, p134

[4] C. Sachrajda, Ref. 1, pl14

[5] Nucl. Phys. B (Proc. Suppl.) 7A (1989)

[6] These proceedings

[7] S. Güsken, U. Löw, K.-H. Mütter, F. Sömmer, A. Patel and K. Schilling, Phys. Lett. 227B (1989) 266

[8] T. Draper, R.M. Woloshyn and K.-F. Iiu, TR.I-PP-8.9-61 (7/8.9)

[9] G. Corbò, E. Franco and G.C. Rossi, Phys. Lett. 221B (1989) 367 
[10] S. Aoki and A. Gocksch, Phys. Rev. Lett. 63 (1989) 1125

[11] Ph. Boucaud, O. Pène, V.J. Hill, C.T. Sachrajda and G. Martinelli, Phys. Lett. 220B (1989) 219

[12] C. Bernard, A. El-Khadra and A. Soni, Ref. 1, p 186

[13] M. Crisafulli, G. Martinelli, V.J. Hill and C.T. Sachrajda, Phys. Lett. 223B (1989) 90

[14] G.W. Kilcup, S.R. Sharpe, R. Gupta and A. Patel, UW 40423-10 P9

[15] J. Gasser and H. Leutwyler, Nucl. Phys. B250 (1985) 465

[16] A. Morel, J. Physique 18 (1987) 1111

[17] E. Eichten, Nucl. Phys. B (Proc. Suppl.) 4 (1988) 170; P. Lepage and B. Thacker, ibid 199

[18] Ph. Boucaud, L.C. Lung and O. Pène, LPTHE-ORSAY 89/01

[19] E. Eichten and B. Hill, FERMILAB-PUB-89/184-T and 89/209-T

[20] M. Bochicchio, L. Maiani, G. Martinelli, G.C. Rossi and M. Testa, Nucl. Phys. B262 (1985) 331

[21] M.B. Voloshin, ITEP 89-13

[22] J. Gasser and H. Leutwyler, Nucl. Phys. B250 (1985) 517

[23] I.I. Bigi, Ref. 5, p 318

[24] S.K. Sharpe, Ref. 5, p 255

[25] M. Gavela, L. Maiani. S. Petrarca, R. Rapuano, G. Martinelli, O .Pène and C. Sachrajda, Nucl. Phys. B306 (1988) 677

[26] S. Sharpe, in preparation

[27] J. Gasser and H. Leutwyler, Phys. Lett. 184B (1987) 83

[28] N. Isgur, K. Maltman, J. Weinstein and T. Rarnes, UTPT-89-10

[29] M. Luscher, Comm. Math. Phys. 105 (1986) 153

[30] J. Donoghue, E. Golowich and B. Holstein, Phys. Lett. 119B (1982) 412

[31] W.A. Bardeen, A.J. Buras and J.-M. Gérard, Phys. Lett. 192B (1987) 138

[32] S.R. Sharpe, R. Gupta, G. Guralnik, G.W. Kilcup and A. Patel, Phys. Lett. 192B (1987) 149 